\title{
New Propagation Effects in Semiconductors in the UV Range: Inhibition of Absorption, Negative Refraction, Anomalous Momentum States, Sub-Wavelength Imaging, and Non-Plamonic Nanometer-size Guided Waves
}

\author{
M. Scalora ${ }^{1}$, M.A. Vincenti ${ }^{1,2}$, V. Roppo ${ }^{1,3}$, John V. Foreman ${ }^{1,4}$, \\ Joseph W. Haus ${ }^{5}$, Neset Akozbek ${ }^{1}$, M.J. Bloemer ${ }^{1}$ \\ ${ }^{1}$ Charles M. Bowden Research Center, AMSRD-AMR-WS-ST, RDECOM, Redstone Arsenal, \\ Alabama 35898-5000,USA \\ ${ }^{2}$ Dipartimento di Elettrotecnica e Elettronica Politecnico di Bari, Via Orabona 4, 70125 Bari Italy \\ ${ }^{3}$ Departament de Fisica i Enginyeria Nuclear, Universitat Politecnica de Catalunya C/Colom 11, \\ 08222 Terrassa Spain \\ ${ }^{4}$ Department of Physics, Duke University, Durham, NC 27708 \\ ${ }^{5}$ Electro-Optics Program, University of Dayton, Dayton, OH 45469-0245, USA
}

\begin{abstract}
We discuss new propagation effects in semiconductors at frequencies above the absorption including inhibition of linear absorption using phase-locked harmonic pulses, negative refraction, anomalous momentum states, sub-wavelength imaging and ultrathin, nanometer-size guiding channels.
\end{abstract}

\section{Introduction}

The primary reason to investigate second harmonic generation (SHG) has always been the achievement of efficient frequency doubling, with emphasis on phase-matched interactions in materials transparent to the second harmonic [1]. In bulk media, phase matching is a momentum-conserving condition that does not occur naturally. Nonlinear conversion efficiencies depend on factors like phase and group velocity mismatch, and pump intensity. Linear absorption at the harmonic frequencies is considered detrimental since it is assumed that the generated harmonics are reabsorbed. In ordinary, positive index materials the generated second-harmonic $(\mathrm{SH})$ signal consists of two components, which are solutions of the homogenous and inhomogeneous wave equations. In a recent study [2] it was noted that in the pulsed regime, the SH signal is characterized by a pulse that is phase-locked and trapped by the pump pulse, and propagates under the pump envelope at the pump's group velocity. In the absence of absorption, the second pulse decouples from the pump and propagates with the characteristic group velocity of the second-harmonic frequency, and obeys material dispersion.

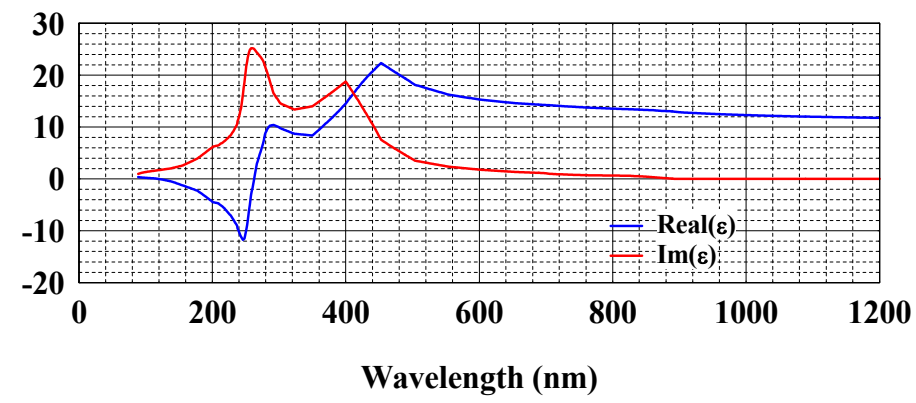

Fig.1: Complex dielectric permittivity of GaAs. The real part becomes negative be low $260 \mathrm{~nm}$.

In Fig. 1 we show the complex dielectric permittivity of GaAs as a function of wavelength. The absorption edge of bulk GaAs occurs around $890 \mathrm{~nm}$. A systematic examination of propagation phenomena, including nonlinear frequency conversion, at frequencies above the absorption edge of semiconductors has not been entertained before primarily because these processes are thought to be unin- 
teresting and inefficient, due to the large imaginary part of the index of refraction, and to the naturally high degree of phase mismatch. We theoretically predict and experimentally observe the inhibition of absorption for pulsed second and third harmonic $(650 \mathrm{~nm}$ and $435 \mathrm{~nm}$, respectively) signals generated in a GaAs substrate 450 microns thick. The characteristic absorption length of GaAs is typically a few tens of nanometers. For instance, transmittance through one micron of GaAs is $\sim 10^{-4}$ at $532 \mathrm{~nm}$, and less than $10^{-8}$ at $364 \mathrm{~nm}$. A pump tuned to $1300 \mathrm{~nm}$ captures and impresses its dispersive properties on portions of the generated second and third harmonic signals, which in turn behave as parts of the pump and co-propagate with the pump pulse for the entire length of the sample without being absorbed [3]. This behavior is brought about by the phase locking phenomenon, which persists regardless of material dispersion. In Fig. 2 we show the fields spectra at the samples' exit.

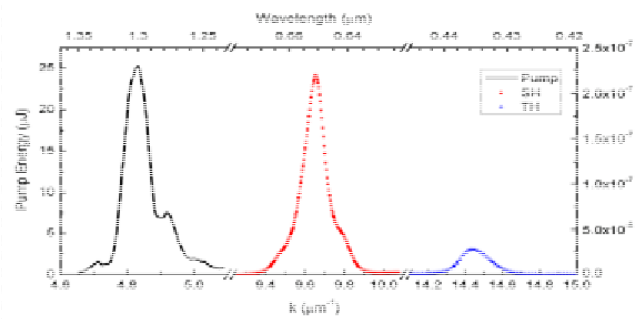

Fig.2 Measured spectra of the pump and the transmitted harmonics. The area under each spectrum corresponds to the measured energy ( $2.9 \mu \mathrm{J}$ for pump, $3.7 \times 10^{-8} \mu \mathrm{J}$ for $\mathrm{SH}, 7.3 \times 10^{-9} \mu \mathrm{J}$ for $\mathrm{TH}$ ).

In semiconductors, the wavelength range of interest is thought to be inaccessible for two reasons: (i) the magnetic permeability is $\mu=1$, and the semiconductor is metallic in nature: no propagating modes are allowed; (ii) absorption is large due to the proximity of resonances (see Fig.1). These properties are very similar to the properties of metals (e.g. silver) below the plasma frequency, which allows a TM-polarized beam to refract negatively inside it. The real part of the dielectric constant of GaAs becomes negative for $\lambda<260 \mathrm{~nm}$, thus making it possible to observe negative refraction for wavelengths below $260 \mathrm{~nm}$. Thus, in analogy to what occurs in resonant metallo-dielectric stacks, a semiconductor-dielectric stack displays negative refraction and resolution below the diffraction limit.

We will also show that the phase-locking mechanism discussed above is capable of producing phase locked harmonic pulses in the regime of negative dielectric permittivity. By tuning a pump pulse in the transparency range, we theoretically predict harmonic generation of phase-locked harmonic pulses in the UV regime, where $\varepsilon<0$, that display anomalous momentum states and undergo negative refraction [3]. The generated harmonic pulses survive over long distances because they acquire the dispersion of the pump, thus inhibiting absorption.

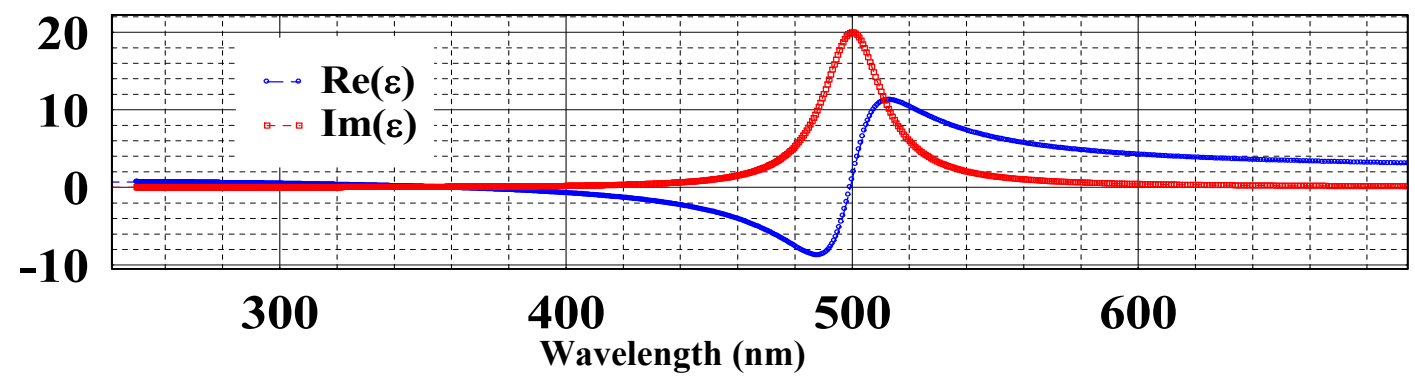

Fig.3: Complex dielectric function for generic Lorentz medium. The pump is tuned above $600 \mathrm{~nm}$.

In addition to negative refraction, I will show that phase locking phenomenon leads to novel kinds of waveguide, where the fields can remain localized inside channels only a few nanometers wide.

[1] P.A. Franken et al, PRL 7, 118 (1961); J. A. Giordmaine, PRL 8, 19 (1962); P.D. Maker et al, PRL 8, 21 (1962); J. A. Armstrong et al, Phys. Rev. 127, 1918 (1962); N. Bloembergen and P. S. Pershan, Phys. Rev. 128, 606 (1962).

[2] V. Roppo et al, Phys. Rev. A76, 033829 (2007); V. Roppo et al., Metamaterials 2, 135 (2008).

[3] M. Centini et al, Phys. Rev. Lett. 101, 113105 (2008). 